\title{
OCURRENCIA DE CARACTERES ANATÓMICOS FUNCIONALES FOLIARES Y CAULINARES EN 35 ESPECIES XERO-HALÓFILAS
}

\author{
VANESA PÉREZ CUADRA ${ }^{1,2}$ y VIVIANA N. CAMBI ${ }^{1}$
}

\begin{abstract}
Resumen: La variada vegetación de los salitrales desarrolla estrategias específicas para enfrentar factores limitantes del crecimiento. Para indagar acerca de estos mecanismos adaptativos se estudió la anatomía foliar y caulinar de 35 especies que habitan el Salitral de la Vidriera (Provincia Buenos Aires), utilizando técnicas histológicas tradicionales. Algunas pocas especies presentan cutícula gruesa mientras que en gran parte de las mismas las células epidérmicas tienen paredes engrosadas. La mayoría de las hojas son anfiestomáticas, presentando algunas estomas protegidos. Sólo tres especies poseen glándulas de sal. El tejido esclerenquimático es escaso a nivel foliar, mientras que en los tallos tanto el esclerénquima como el colénquima alcanzan gran desarrollo. En general ambos órganos poseen tejido acuífero. En la anatomía foliar el tipo de mesofilo más frecuente es el isolateral mientras que el dorsiventral, la estructura Kranz y el céntrico resultan menos frecuentes. Tallos de tres especies presentan endodermis. En varias de las especies se observa crecimiento secundario temprano, así como también el desarrollo de un crecimiento secundario atípico por diferenciación del máster cámbium. En este trabajo, que constituye el primer estudio morfoanatómico en salitrales del sudoeste bonaerense, se comprobó que la ocurrencia de caracteres anatómicos funcionales es más diversa en hojas que en tallos.
\end{abstract}

Palabras clave: xero-halófitas, anatomía ecológica, salitrales.

Summary: Foliar and stem anatomical functional traits occurrence in 35 xero-halophytic species. The varied vegetation of the salt marshes develops specific strategies to address factors that limit growth. To inquire about these adaptive mechanisms, leaf and caulinar anatomy was studied in 35 species inhabiting the Salitral de la Vidriera (Prov. Buenos Aires), using traditional histological techniques. A few species have thick cuticle while much of them have thickened walls of epidermal cells. Most leaves are amphistomatic, presenting some species protected stomata. Only three species have salt glands. There is a scarce development of sclerenchymatic tissue at leaf level, while the stems have a great proportion of sclerenchyma and collenchyma. Overall both organs have aqueous tissue. The most commonly type of leaf anatomy is isolateral mesophyll, the dorsiventral, Kranz and central structure are less frequent. Stems of three species have endodermis. Several species develop an early secondary growth, and in some is observed an atypical secondary growth by the differentiation of master cambium. In this work, which is the first morphoanatomical study in southwest salt marshes of Buenos Aires, it was found that the occurrence of functional anatomical characters is more diverse in leaves than in stems.

Key words: xero-halophytes, ecological anatomy, salt marshes.

\section{INTRODUCCIÓN}

Todos los organismos vivos se encuentran en constante interacción con otros de su misma o diferente especie y con factores abióticos como el

1 Lab. Plantas Vasculares. INBIOSUR-Dep. Biología, Bioquímica y Farmacia. Universidad Nacional del Sur, Bahía Blanca, Argentina.

2 Becaria Posdoctoral de CONICET. Email: vperezcuadra@uns.edu.ar clima, el suelo, el agua, etc., es decir su ambiente (Begon et al., 1999; Valladares et al., 2007); estas interacciones resultan muchas veces ser parte de diferentes factores de estrés ambiental (Schulze et al., 2002). La vegetación de los salitrales es muy variada, no sólo en cuanto a la d iversidad taxonómica sino también en su fisonomía. Esta particular flora exhibe además diferentes estrategias para hacer frente a distintos factores limitantes del crecimiento (Ragonese, 1951), por lo que los salitrales son ambientes muy importantes, como 
hábitats de una gran cantidad de especies nativas y endémicas, y como reservorios de genes con utilidades a futuro en el mejoramiento de especies cultivables (Khan \& Duke, 2001; A shraf et al., 2010; Patil et al., 2012).

Es posible postular algunas simples relaciones sueloplanta entre las comunidades vegetales y las condiciones del suelo. Particularmente para los salitrales algunos autores (Verettoni, 1962; Bertness et al., 1992; Krüger \& Peinemann, 1996) entienden a la salinidad como factor estructurador de las comunidades vegetales, ya que los valores de conductividad eléctrica y la abundancia de especies están correlacionados (al decrecer el nivel de salinidad la comunidad se torna florísticamente más compleja). Fuera de los factores abióticos, existen otros como los de manejo que también afectan la distribución de las especies (Bonis et al., 2005). Es por esto que el uso racional y conservacionista de los suelos de estas zonas es una meta importante, aunque para ello deben conocerse las características del ambiente y de la vegetación que se desarrolla sobre ellos (Coleman, 1970; Abbad et al., 2004; Plenchette \& Duponnois, 2005; Ashraf et al., 2010; Patil et al., 2012). Además del estrés salino, la vegetación halófila está sometida a baja disponibilidad de agua (sequía física y fisiológica), radiación intensa, alternancia de temperaturas extremas y baja fertilidad del suelo (Ruthsatz, 1978; Wahid, 2003). Todos estos factores producen cambios en la morfología, anatomía y ultraestructura de las células vegetales, así como también conllevan a modificaciones fisiológicas que afectan los patrones de crecimiento de las plantas (Reinoso et al., 2005; Ashraf et al., 2010). Dentro de las adaptaciones anatómicas pueden citarse: epidermis gruesas o silicificadas, células buliformes que permiten el enrollamiento de las hojas, estomas protegidos, denso indumento lanoso, esclerénquima bien desarrollado y mesofilo con clorénquima en empalizada compacto (Ancibor, 1980, 1981, 1982; Ragonese, 1990; Fahmy, 1997). Otras características adaptativas son las estructuras secretoras de sal y las vías fotosintéticas. Dentro de las primeras, la presencia de tricomas secretores de sal y glándulas de sal han sido citadas particularmente como distintiva para halófitas. Los tricomas glandulares son muy frecuentes en Atriplex L. spp. (Chenopodiaceae) y en algunos representantes de la familia Poaceae mientras que las glándulas de sal se presentan en Aveniaceae, Acanthaceae, Frankeniaceae, Plumbaginaceae, Poaceae y
Tamaricaceae, aunque sólo algunos géneros de cada una las poseen (Wahid, 2003). Por otro lado, las rutas fotosintéticas adquieren un rol crucial cuando el agua es limitante: las plantas que poseen una vía fotosintética $\mathrm{C}_{4}$ hacen un uso más eficiente del agua que las que utilizan la vía $\mathrm{C}_{3}$ (Kocacinar \& $\mathrm{S}$ age, 2003).

El objetivo de este estudio fue describir la anatomía foliar y caulinar de 35 especies vegetales que crecen espontáneamente en un salitral natural (Salitral de la Vidriera) a $\mathrm{f}$ in de considerar sus estrategias adaptativas al ambiente y evaluar su correspondencia con las ya descriptas para otras especies xero-halófilas.

\section{MATERIALES Y MÉTOdos}

\section{I. Área de estudio}

El área de estudio, Salitral de la Vidriera y su zona aledaña, está ubicada entre los $38^{\circ} 46^{\prime}$ y $38^{\circ} 52^{\prime}$ de latitud sur y $62^{\circ} 34^{\prime}$ y $62^{\circ} 20^{\prime}$ de longitud oeste. Se encuentra situada aproximadamente a 30 kilómetros de la ciudad de Bahía Blanca, Partido de Villarino, Prov. de Buenos Aires, y corresponde a la provincia fitogeográfica del Espinal dentro del distrito del Caldén (Cabrera, 1971). Las precipitaciones anuales oscilan comúnmente entre 400 y $500 \mathrm{~m} \mathrm{~m}$, rangos considerados de ambientes secos. La salinidad en la zona de estudio varía entre $13 \mathrm{dS} . \mathrm{m}^{-1}$ en la zona de menor salinidad y $90 \mathrm{dS} . \mathrm{m}^{-1}$ en la de mayor. La vegetación en la zona se encuentra distribuida en parches (Benedetti et al., 2010) y la flora está constituida por aproximadamente 161 e species vegetales correspondientes a 35 familias botánicas (C. B. Villamil, com. pers.), predominando las especies endémicas y nativas.

\section{Muestreo de especies vegetales}

El muestreo de las especies vegetales fue de tipo mixto, con especies colectadas a través de transectas de $10 \mathrm{~m}$ trazadas desde un camino de acceso y otras colectadas de manera puntual. Se coleccionaron 35 especies pertenecientes a 15 familias en su mayoría nativas y con un alto porcentaje de endémicas (Tabla 1). Los ejemplares de referencia de las especies fueron depositados en el Herbario del Departamento de Biología, Bioquímica y Farmacia de la Universidad Nacional del Sur, Bahía Blanca, Rep. Argentina (BBB) (Thiers, continuously updated). 


\section{Pérez Cuadra y V. N. Cambi - Anatomía foliar y caulinar de xero-halófilas}

Tanto las familias botánicas como las especies estudiadas siguen la taxonomía propuesta por Zuloaga et al. (2008).

\section{Tratamiento del material vegetal para los estudios anatómicos}

De cada especie vegetal se recolectaron hojas y tallos. El material coleccionado fue fijado y conservado en FAA ( formol-alcohol-ácido acético glacial). Las muestras se deshidrataron en una serie de concentración creciente de alcohol etílico-alcohol butílico terciario, se infiltraron en parafina e incluyeron en Paramat (Johansen, 1940). Para las secciones se utilizó la parte media de las hojas y/o folíolos y el tercero a s exto (en algunos hasta el décimo) entrenudo de los tallos. Se realizaron en ambos órganos cortes transversales (seriados en algunos tallos) y longitudinales de $10 \mu \mathrm{m}$ de espesor, con micrótomo rotativo. Las secciones fueron teñidas con safranina-fast green y montadas con bálsamo de Canadá. Se efectuaron pruebas microquímicas accesorias (reactivo de Lugol, sulfato férrico, azul de cresil y Sudán IV) (D'Ambrogio de Argüeso, 1986) p ara la identificación de diferentes sustancias ergásticas presentes en las muestras.

Se analizaron cinco a diez muestras de cada órgano por cada especie colectada. Se estudió el material en microscopio compuesto y las observaciones se registraron mediante microfotografías digitales. La terminología utilizada en este trabajo corresponde a Metcalfe \& Chalk (1950) y Font Quer (2001).

\section{RESULTADOS}

De las 35 especies estudiadas, dos son áfilas (B. trimera y $S$. perennis).

\section{Hoja}

\section{A. Epidermis}

En la mayoría de las especies la cutícula es delgada; en Allenrolfea patagonica, Chuquiraga erinacea, Frankenia juniperoides, Heterostachys olivascens, H. ritteriana, Nitrophila australis, y Pappophorum phillippianum es más gruesa hacia la cara abaxial (Fig. 1 A). Las células epidérmicas generalmente son isodiamétricas a rectangulares. Particularmente en Sesuvium portulacastrum se observan dos morfologías de células epidérmicas (Fig. 1 B): isodiamétricas (reservantes de agua) y rectangulares (notoriamente más pequeñas que las anteriores). Las células epidérmicas reservantes de agua presentan el doble o triple de la altura de las células epidérmicas restantes. En general, en todas las especies la pared tangencial externa de las células epidérmicas está engrosada, siendo las restantes delgadas (Fig. 1 A, D-G, J). Allenrolfea patagonica, Clematis montevidensis, Cressa truxillensis, Grahamia bracteata, Heliotropium curassavicum, Heterostachys olivascens, $H$. ritteriana, Hymenoxys cabrerae, Nitrophila australis, Pappophorum phillippianum, Solanum tweedianum, Suaeda argentinensis, S. divaricata, S. patagonica y Trichloris crinita presentan papilas (Fig. $1 \mathrm{~A}, \mathrm{C}$ ). Sólo en Cressa truxillensis, Limonium brasiliense y Prosopis strombulifera las células epidérmicas poseen taninos (Fig. $1 \mathrm{~N}$ ). En Heliotropium curassavicum se encuentran litocistos con un cistolito redondeado u ovoide en su interior. Las tres especies de Poaceae estudiadas (Melica argyrea, Pappophorum phillippianum y Trichloris crinita) presentan células buliformes (Fig. 1 C, L); en Pappophorum phillippianum y Trichloris crinita las mismas poseen un leve engrosamiento en la pared tangencial externa. En Melica argyrea se encuentran entre cuatro y seis células buliformes (Fig. 1 L), en Pappophorum phillippianum se observan cuatro a siete (Fig. 1 C) y en Trichloris crinita en general cinco; las últimas dos especies presentan parénquima incoloro asociado a las mencionadas células.

En general las hojas son anfiestomáticas, encontrándose en 23 especies estomas a igual nivel que las células epidérmicas restantes, en ocho pseudo-hundidos (Baccharis tenella, Cyclolepis genistoides, Frankenia pulverulenta, Geoffroea decorticans, Heliotropium curassavicum, Suaeda argentinensis, S. divaricata y S. patagonica) (Fig. 1 E) y en dos se observan estomas pseudo-hundidos en la cara abaxial y a nivel epidérmico en la adaxial (Allenrolfea patagonica y Heterostachys ritteriana). Consideramos estomas pseudo-hundidos a aquellos que presentan la pared tangencial externa de las células oclusivas por debajo del nivel de la similar pared en las restantes epidérmicas, al mismo tiempo que las paredes tangenciales internas de ambos tipos celulares se encuentran al mismo nivel (generándose así una pequeña cámara epiestomática); se restringe el concepto de estomas hundidos para aquellos en los que la pared tangencial externa de las células oclusivas se ubica por debajo del nivel de la pared tangencial interna de las células epidérmicas 
Bol. Soc. Argent. Bot. 49 (3) 2014
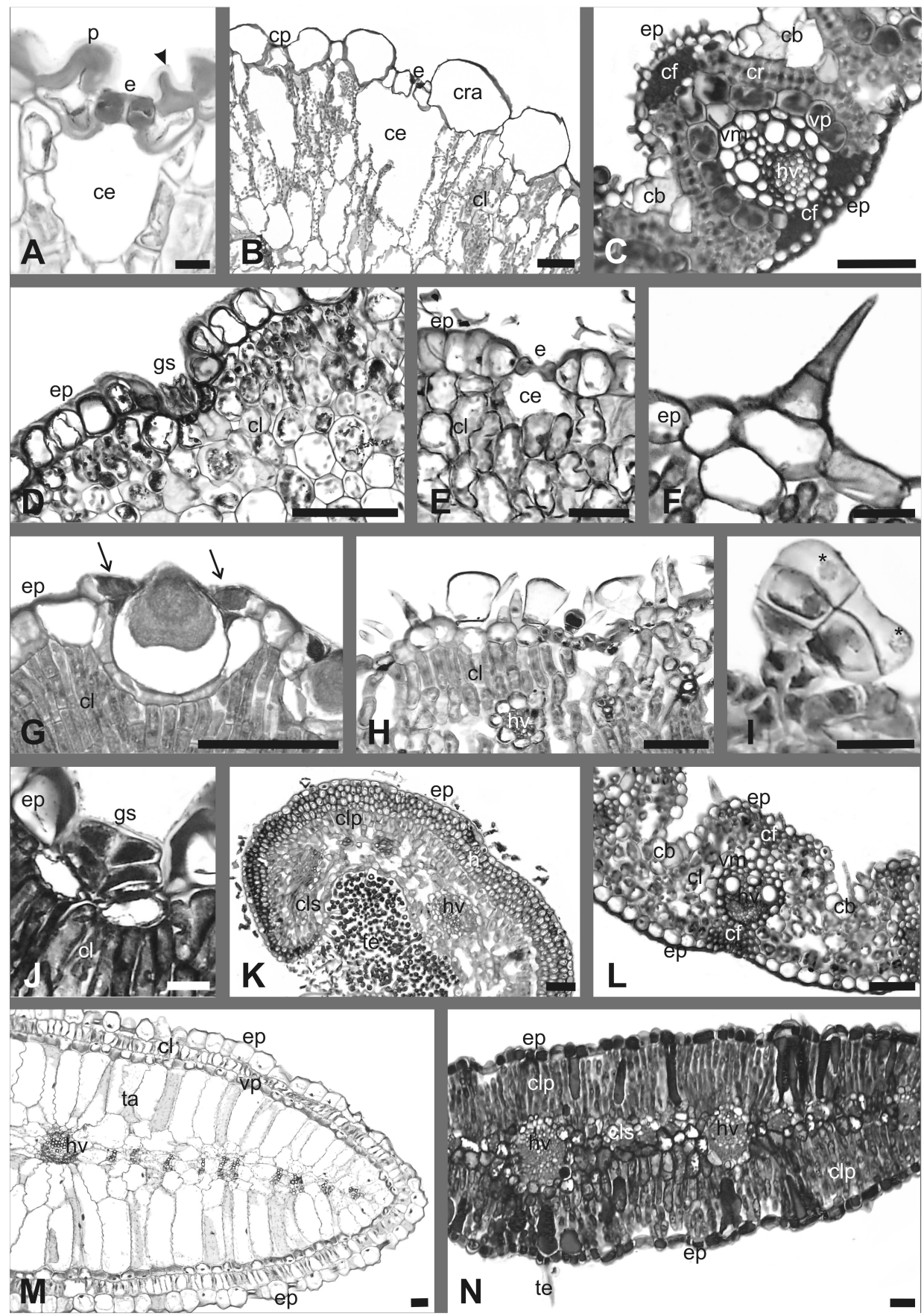

Fig. 1. Caracteres funcionales foliares. A-B, E: Detalle de epidermis y estomas. C, K-N: Estructura del mesofilo. D, J: Detalle de glándulas de sal. F: Detalle de tricoma eglandular. G: Detalle de tricoma cistolítico con litocistos asociados en la base. $\mathrm{H}$ : Detalle de la epidermis con tricomas glandulares y eglandulares. I: Detalle de tricoma glandular con drusas en su célula terminal. A: H. olivascens. B: S. portulacastrum. C: P. phillippianum. D: L. brasiliense. E: C. genistoides. F: G. brachystephana. G-H: A. gratissima. I: B. spartioides. J: F. juniperoides. K: C. erinaceae. L: M. argyrea. M: S. divaricata. N: P. strombulifera. Todos son cortes transversales. Abreviaturas: $\mathrm{cb}$, célula buliforme; ce, cámara subestomática; cf, casquete de fibras; cl, clorénquima; clp, clorénquima en empalizada; cls, clorénquima esponjoso; cra, célula reservante de agua; cr, clorénquima radiado; $\mathrm{cp}$, célula epidérmica; e, estoma; ep, epidermis; gs, glándula de sal; h, hipodermis; hv, haz vascular; p, papila; ta, tejido acuífero; te, tricoma eglandular; vm, vaina mestomática; vp, vaina parenquimática. La cabeza de flecha marca

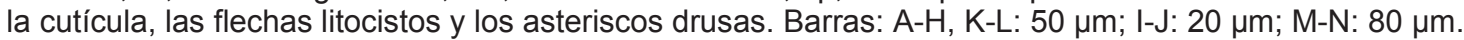




\section{Pérez Cuadra y V. N. Cambi - Anatomía foliar y caulinar de xero-halófilas}

restantes. Algunas especies tienen proyecciones cuticulares en la cara tangencial externa de las células oclusivas. Se observan tricomas eglandulares (Fig. 1 F, H, L) de paredes gruesas y extremo aguzado en Aloysia gratissima, Chuquiraga erinacea, Frankenia juniperoides, Geoffroea decorticans, Grindelia brachystephana, Melica argyrea, Pappophorum phillippianum, Prosopis strombulifera, Schinus sp. y Trichloris crinita, y d e paredes delgadas en Baccharis tenella, Cyclolepis genistoides y Frankenia pulverulenta. En Cressa truxillensis se encuentran tricomas en $\mathrm{T}$ y en Spharealcea australis, tricomas estrellados con las paredes de las células de los brazos esclerosadas mientras que las del pie pueden o n o estarlo. En Aloysia gratissima, además, se observan tricomas eglandulares cistolíticos unicelulares con cistolitos de diferentes tamaños, siempre uno por célula y rodeados por otros litocistos con un solo cistolito en $\mathrm{su}$ interior (Fig. $1 \mathrm{G})$. Se encuentran tricomas glandulares unicelulares (Clematis montevidensis) y multicelulares (Aloysia gratissima, Baccharis spartioides, Cyclolepis genistoides, Grindelia brachystephana, Hymenoxys cabrerae, Senecio pampeanus, Solanum tweedianum y Spharealcea australis) siempre de paredes delgadas (Fig. $1 \mathrm{H}-\mathrm{I}$ ). Los unicelulares son claviformes mientras las cabezas de los multicelulares presentan diferentes morfologías (unicelulares trapezoidales, unicelulares o multicelulares globosas, etc.) (Fig. 1 H-I). En Baccharis spartioides las cabezas de los tricomas glandulares tienen la particularidad de presentar una o dos pequeñas drusas (Fig. 1 I). En Atriplex suberecta, A. undulata, Pappophorum phillippianum y Trichloris crinita se encuentran tricomas secretores de sal; en todos los casos de paredes delgadas. Sólo en Baccharis spartioides los tricomas se encuentran agrupados formando nidos mixtos (tricomas eglandulares y glandulares), mientras que en las restantes especies están dispersos en toda la superficie del órgano. Tres especies presentan glándulas de sal, Frankenia juniperoides, $F$. pulverulenta y Limonium brasiliense. Las dos primeras especies poseen las descriptas como típicas de Frankeniaceae; en Frankenia juniperoides siempre hundidas (Fig. $1 \mathrm{~J}$ ) mientras que en $F$. pulverulenta en algunos casos alcanzan un nivel similar al de las células epidérmicas contiguas. En Limonium brasiliense las glándulas de sal son del tipo característico de las Plumbaginaceae y se ubican frecuentemente en depresiones de la epidermis (Fig. $1 \mathrm{D})$ o al mismo nivel que las células epidérmicas.

\section{B. Mesofilo}

En la zona del nervio medio se halla con frecuencia colénquima angular o paquetes de fibras hacia las caras adaxial o abaxial mientras que en el resto de la lámina sólo las Poaceae presentan fibras subepidérmicas (Fig. 1 C, L). Aloysia gratissima y Atriplex suberecta desarrollan hipodermis unistrata de células parenquimáticas grandes de aspecto turgente. En la primera especie la misma es continua hacia la cara adaxial y abaxial mientras que en la segunda es discontinua y presente sólo hacia la cara adaxial. En Chuquiraga erinacea la hipodermis (solamente abaxial) está formada por cuatro a cinco capas de fibroesclereidas en la región media de la lámina y un número menor de capas hacia los márgenes hasta quedar reducida a una única capa (Fig. $1 \mathrm{~K}$ ). El mesofilo de Geoffroea decorticans se caracteriza por presentar numerosas células taníferas grandes, que en algunos casos alcanzan tamaño similar a 1 os haces vasculares menores. En otras especies, como Limonium brasiliense, se observan taninos dentro de las células del clorénquima. En Allenrolfea patagonica, Baccharis tenella, Cyclolepis genistoides, Heliotropium curassavicum, Heterostachys olivascens, $H$. ritteriana, Hymenoxys cabrerae, Grahamia bracteata, Nitrophila australis, Senecio pampeanus, Sesuvium portulacastrum, Suaeda argentinensis, $S$. divaricata y $S$. patagonica se encontró tejido acuífero, con diferente grado de desarrollo (Fig. 1 M). Los tipos de estructura foliar encontrados son: difuso (Melica argyrea) (Fig. 1 L), dorsiventral inverso (Allenrolfea patagonica, Chuquiraga erinacea, Heterostachys olivascens y $H$. ritteriana) (Fig. $1 \mathrm{~K}$ ), céntrico (Baccharis spartioides, Grahamia bracteata, Hymenoxys cabrerae, Nitrophila australis, Sesuvium portulacastrum y Suaeda patagonica), dorsiventral 
Bol. Soc. Argent. Bot. 49 (3) 2014
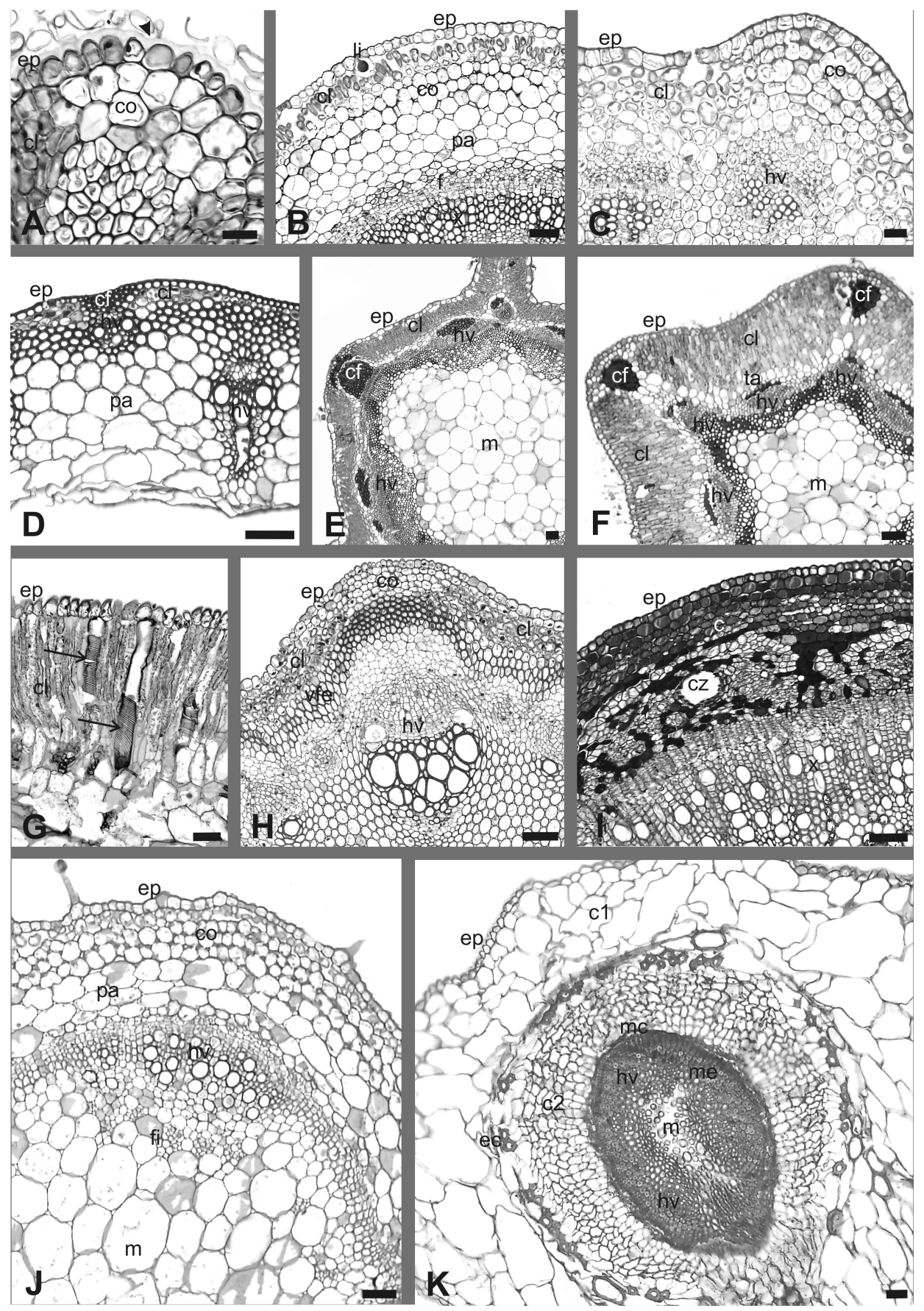

Fig. 2. Caracteres funcionales caulinares. A: Detalle de la epidermis. B-D, H-I: Detalle de la corteza. E-F, J-K: Vista de corteza y estela. G: Detalle de traqueidas corticales. A: C. genistoides. B: H. curassavicum. C: S. pampeanus. D: M. argyrea. E: B. trímera. F: B. tenella. G: S. perennis. H: C. montevidensis. I: Schinus sp. J: S. tweedianum. K: H. ritteriana. Todos son cortes transversales. Abreviaturas: c, corteza; c1, corteza primaria; c2, corteza secundaria; cf, casquete de fibras; cl, clorénquima; co, colénquima; cz, conducto esquizógeno; ec, esclereidas cortizales; ep, epidermis; f, floema; hv, haz vascular; li, litocisto; m, médula; mc, máster cámbium; me, matriz de esclereidas; pa, parénquima; ta, tejido acuífero; vfe, vaina de fibroesclereidas; $x$, xilema. La cabeza de flecha indica la cutícula. Las flechas marcan las traqueidas. Barras: A-K: $50 \mu \mathrm{m}$. 


\section{Pérez Cuadra y V. N. Cambi - Anatomía foliar y caulinar de xero-halófilas}

(Clematis montevidensis, Frankenia juniperoides, F. pulverulenta, Senecio pampeanus, Solanum tweedianum y Spharealcea australis), Kranz (Atriplex suberecta, A. undulata, Pappophorum phillippianum, Suaeda argentinensis, S. divaricata y Trichloris crinita) (Fig. 1 C, M) e isolateral (Aloysia gratissima, Baccharis tenella, Cressa truxilliensis, Cyclolepis genistoides, Geoffroea decorticans, Grindelia brachystephana, Heliotropium curassavicum, Limonium brasiliense, Prosopis strombulifera y Schinus sp.) (Fig. 1 N). Los haces vasculares son colaterales en todas las especies a excepción de los bicolaterales de Solanum tweedianum. Algunos haces vasculares presentan casquetes de colénquima o fibras mientras otros no los poseen. En las tres especies de Poaceae se observan haces vasculares semitrabados (Fig. $1 \mathrm{C}$, L) y libres, y sólo en una de ellas (Trichloris crinita) algunos trabados. En algunas especies se observan vainas parenquimáticas completas (Fig. 1 M) o incompletas, mientras que las restantes no las poseen. Los haces vasculares de Melica argyrea poseen una $\mathrm{v}$ aina mestomática $\mathrm{y}$ parenquimática (Fig. 1 L ) mientras que en Pappophorum phillippianum y Trichloris crinita alternan haces con ambas vainas (Fig. 1 C) y haces sólo con vaina parenquimática. En algunos haces vasculares por fuera del floema, y hacia abaxial de este, se encuentran canales esquizógenos (Baccharis spartioides, B. tenella, Grindelia brachystephana, Schinus sp. y Senecio pampeanus).

\section{Tallo}

\section{A. Epidermis}

La cutícula en general es delgada, a excepción de la presente en Baccharis tenella, Chuquiraga erinacea, Cyclolepis genistoides y Suaeda patagonica (Fig. 2 A). Al igual que en la hoja, en la mayoría de las especies las células epidérmicas presentan la pared tangencial externa engrosada (Fig. 2 A, C-D). Las morfologías más frecuentes de células epidérmicas son: isodiamétricas globosas en Chuquiraga erinacea, Clematis montevidensis, Cyclolepis genistoides, Heliotropium curassavicum, Melica argyrea, Prosopis strombulifera y Suaeda divaricata (Fig. 2 A-B, D, H), isodiamétricas cuadrangulares en Aloysia gratissima, Frankenia juniperoides, Geoffroea decorticans, Hymenoxys cabrerae, Schinus sp., Solanum tweedianum, Spharealcea australis, Suaeda argentinensis y S. patagonica (Fig. $2 \mathrm{~J})$, rectangulares en Atriplex suberecta, $A$. undulata, Baccharis trimera, Cressa truxillensis, Grahamia bracteata, Grindelia brachystephana y Senecio pampeanus (Fig. 2 C) y piriformes en Nitrophila australis y Sarcocornia perennis (Fig. 2 G). Si bien generalmente se observa un tipo morfológico, en algunas especies se encuentran dos: en Sesuvium portulacastrum isodiamétricas y rectangulares (de características similares a las descriptas para la hoja), en Baccharis spartioides sobre las costillas son cuadrangulares a rectangulares y sobre los valles piriformes, en Baccharis tenella y Frankenia pulverulenta las que se encuentran sobre las costillas son piriformes a cuadrangulares y sobre los valles son sólo cuadrangulares. Algunas especies, como Baccharis spartioides, B. tenella, Frankenia pulverulenta, Grindelia brachystephana, Heliotropium curassavicum, Schinus sp., Senecio pampeanus, Solanum tweedianum, Spharealcea australis, Suaeda argentinensis y $S$. divaricata presentan papilas (Fig. 2 B, J). Al igual que en la hoja, en Limonium brasiliense, las células epidérmicas contienen taninos y en Heliotropium curassavicum se hallan litocistos que, por su desarrollo en profundidad, interrumpen prácticamente el clorénquima subepidérmico (Fig. 2 B ). En la mayoría de las especies los estomas se encuentran a nivel de las células epidérmicas, en algunas pseudohundidos (Baccharis tenella, Clematis montevidensis, Cressa truxillensis, Cyclolepis genistoides, Frankenia pulverulenta, Limonium brasiliense y Sarcocornia perennis) y sobreelevados sólo en Aloysia gratissima. Al igual que en las hojas, algunas especies poseen proyecciones cuticulares hacia la cara tangencial externa e interna de las células oclusivas. En 14 especies se encuentran tricomas eglandulares: de paredes delgadas en Baccharis spartioides, $B$. tenella, Cyclolepis genistoides, Frankenia pulverulenta, Grindelia brachystephana y Suaeda argentinensis, y engrosadas en Baccharis trimera, Chuquiraga erinacea, Cressa truxillensis, Frankenia juniperoides, Geoffroea decorticans, Pappophorum phillippianum, Prosopis strombulifera, y Spharealcea australis. Se observaron aguijones (de paredes engrosadas) únicamente en Melica argyrea. Tricomas glandulares se hallaron en 10 especies, y presentan características similares a las descriptas para los foliares. En la cabeza de los tricomas glandulares de dos especies se observan cristales de oxalato de calcio: en Baccharis spartioides pequeñas drusas mientras que en Cyclolepis genistoides arenas 
cristalinas. Sólo en Frankenia pulverulenta y Limonium brasiliense se hallaron glándulas de sal, en cada caso similares a las mencionadas para las hojas. En Frankenia pulverulenta se ubican a nivel epidérmico o levemente hundidas mientras que en Limonium brasiliense se ubican sólo a $\mathrm{n}$ ivel epidérmico.

\section{B. Corteza}

En Aloysia gratissima, Baccharis spartioides, B. tenella, B. trimera, Clematis montevidensis, Cressa truxillensis, Cyclolepis genistoides, Frankenia pulverulenta, Geoffroea decorticans, Heliotropium curassavicum, Hymenoxys cabrerae, Melica argyrea, Pappophorum phillippianum, Sarcocornia perennis, Senecio pampeanus, Sesuvium portulacastrum, Solanum tweedianum y Spharealcea australis, se observa clorénquima subepidérmico (Fig. 2 B -C, E-H). En Baccharis spartioides, Clematis montevidensis, Hymenoxys cabrerae y Senecio pampeanus el clorénquima alterna con colénquima angular (Fig. 2 H) y en Melica argyrea, Pappophorum phillippianum y Trichloris crinita, con paquetes de fibras (Fig. 2 D). Particularmente en Heliotropium curassavicum, Solanum tweedianum y Spharealcea australis debajo del clorénquima se encuentra colénquima, angular en la primera especie (Fig. 2 B) y lagunar en las otras dos (Fig. $2 \mathrm{~J}$ ). En Aloysia gratissima el clorénquima se encuentra por debajo de una capa de colénquima angular. En Baccharis tenella y B. trimera en las zonas costales a nivel subepidérmico se encuentran paquetes de fibras (Fig. 2 E-F) y en Aloysia gratissima y Grahamia bracteata se diferencian paquetes de fibroesclereidas; en las zonas intercostales se encuentra clorénquima acompañado, o no, de colénquima. En cinco especies se encuentra tejido acuífero, con una distribución particular en Baccharis tenella a modo de vaina incompleta rodeando al clorénquima por ambos lados y hacia el interior (Fig. 2 F). En Sarcocornia perennis, tanto en el clorénquima como en el tejido acuífero, se encuentran traqueidas grandes y aisladas con depósito espiralado de pared secundaria (Fig. 2 G). En Frankenia juniperoides, Limonium brasiliense, Prosopis strombulifera y Schinus sp., las células parenquimáticas de la corteza tienen gran cantidad de taninos (Fig. 2 I), mientras que en Geoffroea decorticans se encuentran células tánicas dispersas. En Baccharis spartioides en posición cortical más profunda, y en general en relación a los valles, se desarrollan canales esquizógenos de gran tamaño. En Clematis montevidensis y Prosopis strombulifera se encuentran fibras, acompañadas de fibroesclereidas en la segunda especie, rodeando la estela (Fig. 2 H). En Cressa truxillensis y Frankenia juniperoides, también en una posición profunda en la corteza, se observa una capa de esclereidas corticales, formando un anillo completo en la segunda especie e incompleto en la p rimera. Baccharis tenella, B. trimera y Sarcocornia perennis presentan endodermis.

\section{Estela}

Los tejidos conductores de los haces vasculares primarios tienen disposición colateral en todas las especies (Fig. $2 \mathrm{E}-\mathrm{F}, \mathrm{H}, \mathrm{J}$ ). Particularmente en Limonium brasiliense, Pappophorum phillipianum y Trichloris crinita los haces vasculares forman anillos concéntricos. En Clematis montevidensis se observan entre los haces vasculares igual cantidad de paquetes de floema (Fig. 2 H). En Solanum tweedianum se encuentra floema intraxilar enfrentado a los polos protoxilemáticos de los haces vasculares (Fig. $2 \mathrm{~J}$ ). Algunas especies presentan casquetes de tejido de sostén asociados a los haces vasculares: de fibras floemáticas en Baccharis trimera, Grindelia brachystephana, Heliotropium curassavicum, Hymenoxys cabrerae, Geoffroea decorticans y Spharealcea australis y de tejido colenquimático angular en Nitrophila australis y Sesuvium portulacastrum. En Grindelia brachystephana y Schinus sp. en estrecha relación con el floema, se encuentran conductos esquizógenos (Fig. 2 I). En Limonium brasiliense y Geoffroea decorticans, el parénquima xilemático y floemático posee taninos. En la mayoría de las especies se observó un desarrollo temprano del cámbium vascular y tejidos vasculares secundarios. El crecimiento secundario es principalmente típico (Fig. 2 I) y en algunas especies (Allenrolfea patagonica, Atriplex suberecta, A. undulata, Heterostachys olivascens, $H$. ritteriana, Sarcocornia perennis, Suaeda divaricata y S. patagonica) atípico por acción del máster cambium (Fig. 2 K). En estas últimas especies se diferencia corteza secundaria (formada por células parenquimáticas) y matriz esclerenquimática con haces vasculares secundarios incluidos en ella (Fig. $2 \mathrm{~K}$ ). Se encontraron en general médulas amplias, formadas por células parenquimáticas principalmente de paredes delgadas. Particularmente en Baccharis tenella, B. 


\section{Pérez Cuadra y V. N. Cambi - Anatomía foliar y caulinar de xero-halófilas}

trimera y Spharealcea australis la médula presenta algunas células parenquimáticas con paredes esclerosadas en la zona de contacto con el xilema (Fig. 2 F ). En el parénquima medular se hallaron taninos en algunas especies (Limonium brasiliense, Prosopis strombulifera y Spharealcea australis) y cristales en otras (Grindelia brachystephana y Sesuvium portulacastrum). En Allenrolfea patagonica, Cressa truxillensis, Frankenia pulverulenta, Hymenoxys cabrerae y Sarcocornia perennis las células medulares más internas tienden a romperse generando tallos huecos a la $\mathrm{m}$ adurez (Fig. $2 \mathrm{~K}$ ).

\section{Discusión}

Los caracteres típicos de plantas xerófilas y halófilas ya descriptos, son básicamente: estomas hundidos en criptas, estructuras secretoras de sal e importante desarrollo de tejido esclerenquimático (Bianco et al., 2004; Ashraf et al., 2010). En este estudio no todas estas características fueron encontradas y sí se hallaron otras como: estomas pseudo-hundidos, presencia de tejido acuífero, crecimiento secundario atípico, etc. Por otro lado, y en concomitancia con trabajos anteriores (Bianco et al., 2004; Feijóo et al., 2005; Cutler et al., 2007), comparando la diversidad de características anatómicas entre hojas y tallos, se observó que la may or variabilidad se encuentra a nivel foliar.

La sequedad de la atmósfera es considerada uno de los principales factores que aumenta la producción de cutina (Delf, 1915). Esta sustancia ergástica es una efectiva barrera de protección contra los vientos fuertes y la $\mathrm{r}$ adiación solar que producen una excesiva transpiración (Arambarri et al., 2006). Si bien este carácter suele ser citado como frecuente en xerófitas y halófitas, entre las aquí estudiadas resulta poco común tanto en hojas como en tallos.

Existen dos estrategias protectoras de los estomas para evitar la pérdida excesiva de agua, una referida a su localización en una $u$ otra epidermis y la otra al nivel del estoma en relación a las células epidérmicas circundantes (Ancibor, 1980). Entre las especies aquí estudiadas, pocas poseen los estomas sólo en la cara abaxial de la hoja al igual que las mesófitas (Cutler et al., 2007); mientras que, la mayor parte son de tipo anfiestomáticas característica común entre las xerófitas (Fahmy, 1997). Dentro de la segunda estrategia de protección de los estomas la localización de éstos, hundidos o ubicados en criptas, es considerada un carácter altamente adaptativo a un ambiente seco; en ninguna de las especies estudiadas se encontró este tipo de disposición de los estomas. Sin embargo se observaron algunos no hundidos sensu stricto sino pseudo-hundidos tanto en hojas como en tallos, disposición hasta la presente publicación no descripta en la bibliografía (ver definición en Resultados). Si bien estomas de este tipo fueron encontrados anteriormente en hojas de otras especies de Chenopodiaceae y Myrothamnaceae (Polić et al., 2009; Lavalle \& Mengascini, 2000; K orte \& Porembski, 2012) fueron reportados como hundidos sensu lato.

Los tricomas, principalmente los eglandulares, cuando se encuentran en abundante cantidad retienen una capa de aire sobre la superficie de la hoja que genera un gradiente para ciertos factores condicionantes de la evapotranspiración: humedad y temperatura (Johnson, 1975; Ancibor, 1981; Fahmy, 1997; Dickison, 2000; Cutler et al., 2007; Ward, 2009). Cuando la cantidad de tricomas es insuficiente para generar esa capa de aire, actúan naturalmente de manera inversa aumentando la superficie de la hoja, y por lo tanto, incrementando la superficie expuesta a la evapotranspiración (Johnson, 1975; Dickinson, 2000; Cutler et al., 2007). En estos casos es común que los tricomas esclerosen sus paredes para evitar la pérdida de agua (Johnson, 1975). En las especies aquí estudiadas se encontraron tricomas en gran cantidad de paredes delgadas o esclerosadas o tricomas escasos siempre de paredes esclerosadas, correlacionándose los mismos con las características citadas para el ambiente.

Sólo tres de las especies aquí estudiadas presentaron glándulas de sal, de las cuales dos pertenecen a Frankeniaceae y la otra a Plumbaginaceae, siendo las estructuras de las glándulas las descriptas como típicas para estas familias (Metcalfe \& Chalk, 1950; Salama et al., 1999).

La presencia de gran cantidad de tejido esclerenquimático en las hojas es una característica muy importante como indicador de xeromorfismo (Ragonese, 1990). Este tejido confiere una notable rigidez a los órganos y su abundancia se relaciona con la pobreza del suelo (Ancibor, 1982). En las 
especies estudiadas en este trabajo se encontró un escaso desarrollo de tejido esclerénquimatico foliar, con ausencia, en la mayoría de las plantas, de cualquier tipo de tejido de sostén subepidérmico. Esto puede vincularse con el reducido tamaño de las hojas y el desarrollo del tejido acuífero que les otorga turgencia y sostén mecánico. Por el contrario en los tallos de varias especies se observó gran desarrollo de tejidos de sostén subepidérmico (colénquima y esclerénquima). El tipo de estructura foliar más frecuentemente encontrada en las especies aquí estudiadas es la d e mesofilo isolateral; la estructura dorsiventral, Kranz y céntrica también son frecuentes mientras que la estructura dorsiventral inversa y el clorénquima difuso son poco usuales. La suculencia en hojas y tallos no está relacionada con un grupo taxonómico particular ni con el hábito de crecimiento del vegetal pero existe una convergencia en las estrategias de almacenamiento y uso del agua, que hace que muchas especies parezcan muy similares, habiendo alcanzado este carácter a través de vías evolutivas diferentes (Eggli \& Nyffeler, 2009). Entre las especies aquí estudiadas el tejido acuífero es más abundante en hojas que en tallos, probablemente esta diferencia se relacione con el mayor desarrollo de tejidos de sostén en los tallos. En la mayoría de las especies aquí estudiadas se observó crecimiento secundario temprano, una característica generalmente frecuente en especies de ambientes hostiles ya que otorga mayor rigidez a los tallos y, en muchos casos, protege a los tejidos conductores. Algunas especies presentaron crecimiento secundario originado por máster cambium (Carlquist, 2007). En este tipo de crecimiento secundario los tejidos conductores, sobre todo el floema, se encuentran protegidos por tejidos de mayor resistencia contribuyendo a e vitar su colapso (Fahn \& Shchori, 1967; Baird \& Blackwell, 1980; Fahn \& Zimmermann, 1982, Carlquist, 1995, 2007; Schweingruber, 2007; Polić et al., 2009).

\section{CONCLUSIONES}

Este trabajo constituye el primer estudio morfoanatómico a $\mathrm{n}$ ivel de comunidades vegetales en salitrales del sudoeste bonaerense. A partir del mismo se concluye que la cutícula delgada es un carácter muy frecuente entre las xerófitas y halófitas que habitan el Salitral de la Vidriera y se reporta por primera vez la e xistencia de estomas pseudohundidos. Se descarta la presencia de glándulas de sal como carácter típico de estas plantas xerohalófilas, ya que sólo tres de las treinta y cinco especies presentaron glándulas de sal, destacándose que la especie que tolera la máxima salinidad de la zona (H. ritteriana) no las posee.

\section{BIBLIOGRAFÍA}

ABBAD, A., A. EL HADRAMI, I. EL HADRAMI \& A. BENCHAABANE. 2004. Atriplex halimus (Chenopodiaceae): a halophytic species for restoration and rehabilitation of saline degraded lands. Pakistan J. Biol. Sci. 7: 1085-1093.

ANCIBOR, E. 1980. Estudio anatómico de la vegetación de la Puna de Jujuy. II. Anatomía de las plantas en cojín. Bol. Soc. Argent. Bot. 19: 157-202.

ANCIBOR, E. 1981. Estudio anatómico de la vegetación de la Puna de Jujuy. III. Anatomía de las plantas en roseta. Lilloa 3: 125-136.

ANCIBOR, E. 1982. Estudio anatómico de la vegetación de la Puna de Jujuy. IV. Anatomía de los subarbustos. Physis 41: 107-114.

ARAMBARRI, A. M., S. E. FREIRE, M. N. COLARES, N. D. BAYÓN, M. C. NOVOA, C. MONTI \& S. A. STENGLEIN. 2006. Leaf anatomy of medicinal shrubs and tres from gallery forests of the Paranaense Province (Argentina). Part I. Bol. Soc. Argent. Bot. 41: 233-268.

ASHRAF, M., M. OZTURK \& M. S. A. AHMAD. 2010. Plant adaptation and phytoremediation. Springer, New York.

BAIRD, W. V. \& W. H. BLACKWELL. 1980. Secondary growth in the axis of Halogeton glomeratus (Bieb.) Meyer (Chenopodiaceae). Bot. Gaz. 141: 269-276.

BEGON, M., J. L. HARPER \& C. R. TOWNSEND. 1999. Ecología: individuos, poblaciones y comunidades. Ediciones Omega, Barcelona.

BENEDETTI, G., A. CAMPO \& A. GERALDI. 2010. Las nuevas tecnologías aplicadas a la ecología del paisaje: estudio de un área del 


\section{Pérez Cuadra y V. N. Cambi - Anatomía foliar y caulinar de xero-halófilas}

Salitral de la V idriera, Provincia de Buenos Aires. GeoSIG 2: 126-134.

BERTNESS, M. D., L. GOUGH \& S. W. SHUMWAY. 1992. Salt tolerances and the distribution of fugitive salt marsh plants. Ecology 73: 1842-1851.

BIANCO, C. A., T. A. KRAUS \& A. C. VEGETTI. 2004. La hoja: morfologia y anatomia. Universidad Nacional de Río Cuarto, Córdoba.

BONIS, A., J. B. BOUZILlÉ, B. AMIAUD \& G. LOUCOUGARAY. 2005. Plant community patterns in old embanked grasslands and the survival of halophytic flora. Flora 200: 74-87.

CABRERA, A. L. 1971. Fitogeografía de la República Argentina. Bol. Soc. Argent. Bot. 14: $1-48$.

CARLQUIST, S. 1995. Wood anatomy of Caryophyllaceae: ecological, habital, systematic and phylogenetic implications. Aliso 14: $1-17$.

CARLQUIST, S. 2007. Succesive cambia revisited: ontogeny, histology, diversity, and functional significance. J. Torrey Bot. Soc. 134: 301-332.

COLEMAN, A. M. 1970. The conservation of wildscape: a q uest for facts. Geogr. J. 136: 199-205.

CUTLER, D. F., T. BOTHA \& D. W. STEVENSON. 2007. Plant anatomy, an applied approach. Blackwell Publ., Singapur.

D'AMBROGIO DE ARGÜESO, A. 1986. Manual de técnicas en histología vegetal. Hemisferio Sur, Buenos Aires.

DELF, E. M. 1915. The meaning of xerophily. J. Ecol. 3: 110-121.

DICKISON, W. C. 2000. Integrative Plant Anatomy. Academic Press, San Diego.

EGGLI, U. \& R. NYFFELER. 2009. Living under temporarily arid conditions-succulence as an adaptive strategy. Bradleya 27: 13-36.

FAHMY, G. M. 1997. Leaf anatomy and its relation to the ecophysiology of some non-succulent desert plants from Egypt. J. Arid Environ. 36: 499-525.

FAHN, A. \& Y. SHCHORI. 1967. The organization of the secondary conducting tissues in some species of the Chenopodiaceae. Phytomorphology 17: 147-154.
FAHN, A. \& M. H. ZIMMERMANN. 1982. Development of the successive cambia in Atriplex halimus (Chenopodiaceae). Bot. Gaz. 143: 353-357.

FEIJÓO, M. S., M. E. ARCE \& A. D'AMBROGIO. 2005. Variación morfoanatómica en una población de Senecio filaginoides (Asteraceae). Polibotánica 19: 1-17.

FONT QUER, P. 2001. Diccionario de botánica. Ediciones Península, Barcelona.

JOHANSEN, D. A. 1940. Plant microtechnique. Mc Graw-Hill, New York.

JOHNSON, H. 1975. Plant pubescense: an ecological perspective. Bot. Rev. 41: 233-258.

KHAN, M. A. \& N. C. DUKE. 2001. Halophytes A resource for the future. Wetlands Ecol. Manag. 6: 455-456.

KOCACINAR, F. \& R. F. SAGE. 2003. Photosybthetic pathway alters xylem structure and hydraulic function in herbaceous plants. Plant Cell Environ. 26: 2015-2026.

KORTE, N. \& S. POREMBSKI. 2012. A morphoanatomical characterisation of Myrothamnus moschatus (Myrothamnaceae) under the aspect of desiccation tolerance. Plant Biol. 14: 537 541.

KRÜGER, H. R. \& N. PEINEMANN. 1996. Coastal plain halophytes and their relation to soil ionic composition. Vegetatio 122: 143-150.

LAVALLE, M. C. \& A. MENGASCINI. 2000. Estomatogénesis y estomas adultos en Suaeda divaricata Moq.-Tand. (Chenopodiaceae). Revista Museo La Plata, Bot. 14: 471-476.

METCALFE, C. R. \& L. CHALK. 1950. Anatomy of the Dicotyledons; leaves, stem and wood in relation to taxonomy with notes on economic uses. Clarendon Press, Oxford.

PATIL, A. V., V. H. LOKHANDE, P. SUPRASANNA, V. A. BAPAT \& J. P. JADHAV. 2012. Sesuvium portulacastrum (L.) L.: a potential halophyte for the degradation of toxic textile dye, Green HE4B. Planta 235: 1051-1063.

PLENCHETTE, C. \& R. DUPONNOIS. 2005. Growth response of the saltbush Atriplex nummularia L. to inoculation with the arbuscular mycorrhizal fungus Glomus intraradices. J. Arid Environ. 61: 535-540. 
POLIĆ, D., J. LUKOVIC, L. ZORICं, L. MERKULOV \& A. KNEŽEVIĆ. 2009. Morpho-anatomical differentiation of Suaeda maritima (L.) Dumort. 1827. (Chenopodiaceae) populations from inland and maritime saline area. Centr. Eur. J. Biol. 4: 117-129.

RAGONESE, A. E. 1951. La vegetación de la República Argentina II. Estudio Fitosociológico de las Salinas Grandes. RIA 5: 1-234.

RAGONESE, A. M. 1990. Caracteres xeromorfos foliares de Nassauvia lagascae (Compositae). Darwiniana 30: 1-10.

REINOSO, H., L. SOSA, M. REGINATO \& V. LUNA. 2005. Histological alterations induced by sodium sulfate in the vegetative anatomy of Prosopis stombulifera (Lam.) Benth. Wordl J. Agric. Sci. 1:109-119.

RUTHSATZ, B. 1978. Las plantas en cojín de los semi-desiertos andinos del Noroeste Argentino. Darwiniana 21: 491-539.

SALAMA, F. M., S. M. EL-NAGGAR \& T. RAMADAN. 1999. Salt glands of some halophytes in Egypt. Phyton 39: 91-105.

SCHULZE, E. D., E. BECK \& K. MÜLLER HOHRNSTEIN. 2002. Plant Ecology. Springer, Berlín.
SCHWEINGRUBER, F. H. 2007. Stem anatomy of Caryophyllaceae. Flora 202: 281-292.

THIERS, B. Cotinuously undapted. Index herbariorum: a global directory of public herbaria and associated staff. New York Botanical Garden's Virtual Herbarium. Disponible en: http://sweetgum.nybg.org/ih [Acceso: 19 de mayo 2014].

VALLADARES, F., E. GIANOLI \& J. M. GÓMEZ. 2007. Ecological limits to plant phenotypic plasticity. New Phytol. 176: 749-763.

WAHID, A. 2003. Physiological significance of morpho-anatomical features of the halophytes with particular reference to cholistan flora. Int. J. Agric. Biol. 5: 207-212.

VERETTONI, H. N. 1962. La salinidad de los suelos y el problema biológico y agrícola de las plantas tolerantes a la sal de la zona de Bahía Blanca. Universidad Nacional del Sur, Bahía Blanca.

WARD, D. 2009. The biology of deserts. Oxford University Press, New York.

ZULOAGA, F. O., O. MORRONE \& M. J. BELGRANO. 2008. Catálogo de las Plantas Vasculares del Cono Sur (Argentina, Sur de Brasil, Chile, Paraguay y Uruguay). Missouri Botanical Garden Press, Missouri.

Recibido el 19 de diciembre de 2013, aceptado el 28 de mayo de 2014. 


\section{Pérez Cuadra y V. N. Cambi - Anatomía foliar y caulinar de xero-halófilas}

Tabla 1. Especies estudiadas con detalle de sus estatus para Argentina y material de referencia

\begin{tabular}{|c|c|c|c|}
\hline Familia & Especie & Estatus & $\begin{array}{c}\text { Material de } \\
\text { referencia }\end{array}$ \\
\hline Aizoaceae & Sesuvium portulacastrum (L.) L. & Nativa & $\begin{array}{c}\text { Villamil y } \\
\text { Fernández, } 6878\end{array}$ \\
\hline Anacardiaceae & Schinus sp. & Nativa & Villamil, 10166 \\
\hline \multirow{8}{*}{ Asteraceae } & Baccharis spartioides (Hook. \& Arn. ex DC.) J. Remy & Nativa & Pérez Cuadra, 35 \\
\hline & Baccharis tenella Hook. \& Arn. & Nativa & Pérez Cuadra, 36 \\
\hline & Baccharis trimera (Less.) DC. & Nativa & Pérez Cuadra, 37 \\
\hline & Chuquiraga erinacea D. Don & Endémica & Villamil, 10092 \\
\hline & Cyclolepis genistoides D. Don & Nativa & Villamil, 10170 \\
\hline & Grindelia brachystephana Griseb. & Endémica & Pérez Cuadra, 38 \\
\hline & Hymenoxys cabrerae K. L. Parker & Endémica & Pérez Cuadra, 39 \\
\hline & Senecio pampeanus Cabrera & Nativa & $\begin{array}{c}\text { Villamil y Sánchez, } \\
3214\end{array}$ \\
\hline Boraginaceae & Heliotropium curassavicum L. & Nativa & $\begin{array}{c}\text { Villamil y Sánchez, } \\
3213\end{array}$ \\
\hline \multirow{10}{*}{ Chenopodiaceae } & Allenrolfea patagonica Moq. (Kuntze) & Endémica & $\begin{array}{c}\text { Villamil y Sánchez, } \\
3219\end{array}$ \\
\hline & Atriplex suberecta I Verd. & Adventicia & Villamil, 10138 \\
\hline & Atriplex undulata (Moq.) D. Dietr. & Endémica & Villamil, 10098 \\
\hline & Heterostachys olivascens (Speg.) Speg. & Endémica & Villamil, 10139 \\
\hline & Heterostachys ritteriana (Moq.) Ung.-Sternb. & Nativa & Villamil, 10141 \\
\hline & Nitrophila australis Chodat \& Wilczek & Endémica & $\begin{array}{c}\text { Villamil y Sccofield } \\
10144\end{array}$ \\
\hline & Sarcocornia perennis (Mill.) A. J. Scott & Introducida & Pérez Cuadra, 43 \\
\hline & Suaeda argentinensis A. Soriano & Endémica & Villamil, 10135 \\
\hline & Suaeda divaricata Moq. & Endémica & Villamil, 10158 \\
\hline & Suaeda patagonica Speg. & Endémica & Pérez Cuadra, 47 \\
\hline Convolvulaceae & Cressa truxillensis Kunth & Nativa & Pérez Cuadra, 50 \\
\hline \multirow{2}{*}{ Fabaceae } & Geoffroea decorticans (Gillies ex Hook. \& Arn.) Burkart & Nativa & Pérez Cuadra, 51 \\
\hline & Prosopis strombulifera (Lam.) Benth. & Nativa & Pérez Cuadra, 52 \\
\hline \multirow{2}{*}{ Frankeniaceae } & Frankenia juniperoides (Hieron.) M. N. Correa & Nativa & Pérez Cuadra, 46 \\
\hline & Frankenia pulverulenta L. & Naturalizada & Pérez Cuadra. 45 \\
\hline Malvaceae & Sphaeralcea australis Speg. & Endémica & Pérez Cuadra, 55 \\
\hline Plumbaginaceae & Limonium brasiliense (Boiss.) Kuntze & Nativa & Pérez Cuadra, 58 \\
\hline \multirow{3}{*}{ Poaceae } & Melica argyrea Hack. & Nativa & Pérez Cuadra, 59 \\
\hline & Pappophorum phillippianum Parodi & Nativa & Villamil, 10090 \\
\hline & Trichloris crinita (Lag.) Parodi & Nativa & Pérez Cuadra, 61 \\
\hline Portulacaceae & Grahamia bracteata Hook. \& Arn. & Endémica & $\begin{array}{c}\text { Villamil y } \\
\text { Fernández, } 6874\end{array}$ \\
\hline Ranunculaceae & Clematis montevidensis Spreng. & Nativa & Pérez Cuadra, 63 \\
\hline Solanaceae & Solanum tweedianum Hook. & Endémica & Pérez Cuadra, 65 \\
\hline Verbenaceae & Aloysia gratissima (Gillies \& Hook. ex Hook.) Tronc. & Nativa & Pérez Cuadra, 62 \\
\hline
\end{tabular}

